\title{
Photoluminescence of a quantum-dot molecule
}

Stanislav Yu. Kruchinin, Ivan D. Rukhlenko, Anvar S. Baimuratov', Mikhail Yu. Leonov, Vadim K. Turkov, Yurii K. Gun'ko, Alexander V. Baranov, and Anatoly V. Fedorov

Citation: Journal of Applied Physics 117, 014306 (2015); doi: 10.1063/1.4905258

View online: http://dx.doi.org/10.1063/1.4905258

View Table of Contents: http://aip.scitation.org/toc/jap/117/1

Published by the American Institute of Physics 


\title{
Photoluminescence of a quantum-dot molecule
}

\author{
Stanislav Yu. Kruchinin, ${ }^{1}$ Ivan D. Rukhlenko, ${ }^{2,3}$ Anvar S. Baimuratov, ${ }^{3,2, a)}$ \\ Mikhail Yu. Leonov, ${ }^{3}$ Vadim K. Turkov, ${ }^{3}$ Yurii K. Gun'ko, ${ }^{4,3}$ Alexander V. Baranov, ${ }^{3}$ \\ and Anatoly V. Fedorov ${ }^{3}$ \\ ${ }^{1}$ Max Planck Institute of Quantum Optics, Hans-Kopfermann-Straße 1, D-85748 Garching, Germany \\ ${ }^{2}$ Monash University, Clayton Campus, Victoria 3800, Australia \\ ${ }^{3}$ ITMO University, 197101 Saint Petersburg, Russia \\ ${ }^{4}$ School of Chemistry and CRANN Institute, Trinity College, Dublin, Dublin 2, Ireland
}

(Received 1 November 2014; accepted 18 December 2014; published online 5 January 2015)

\begin{abstract}
The coherent coupling of quantum dots is a sensitive indicator of the energy and phase relaxation processes taking place in the nanostructure components. We formulate a theory of low-temperature, stationary photoluminescence from a quantum-dot molecule composed of two spherical quantum dots whose electronic subsystems are resonantly coupled via the Coulomb interaction. We show that the coupling leads to the hybridization of the first excited states of the quantum dots, manifesting itself as a pair of photoluminescence peaks with intensities and spectral positions strongly dependent on the geometric, material, and relaxation parameters of the quantum-dot molecule. These parameters are explicitly contained in the analytical expression for the photoluminescence differential cross section derived in the paper. The developed theory and expression obtained are essential in interpreting and analyzing spectroscopic data on the secondary emission of coherently coupled quantum systems. (C) 2015 AIP Publishing LLC. [http://dx.doi.org/10.1063/1.4905258]
\end{abstract}

\section{INTRODUCTION}

The nonradiative transfer of energy in low-dimensional structures has been the subject of much research ${ }^{1-4}$ due to the many prospective uses of this phenomenon in optoelectronics, ${ }^{5,6}$ quantum computing, ${ }^{7-9}$ biology, and medicine. ${ }^{10,11}$ The first theory of nonradiative energy transfer was developed in the late 1940 s by Förster, ${ }^{12}$ who studied the resonant migration of energy between a pair of dye molecules using the semiclassical quantum approach, while considering the dipole-dipole interaction between the molecules. Förster's theory was extended a few years later by Dexter ${ }^{13}$ to include transfer by means of dipole-forbidden transitions, which occur due to the overlapping of the dipole field of a sensitizer with the quadrupole field of an activator and exchange effects. The past decade has witnessed the emergence of many experimental ${ }^{14-17}$ and theoretical ${ }^{18-25}$ works devoted to the investigation of various aspects of nonradiative energy exchange between semiconductor quantum dots (QDs), including the studies on how the exchange is affected by the nearby metallic nanoparticles ${ }^{26,27}$ and photon modes of optical microcavities. ${ }^{28,29}$ The widespread interest in different kinds of QD nanostructures_-including molecules and oligomers, ${ }^{30,31}$ two- and three-dimensional supercrystals, ${ }^{32-35}$ as well as dendrites ${ }^{36,37}$ - is explained by the sizedependent energy spectrum of QDs, their high chemical stability, and fluorescence brightness (the product of the quantum yield and extinction coefficient). These features make QD nanostructures ideal objects for experimental studies of nonradiative energy transfer via the methods of optical spectroscopy. Of significance from the theoretical viewpoint

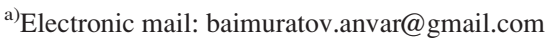

is that in many practical instances such transfer can be adequately described within the framework of the dipole-dipole approximation even when the QDs almost touch each other. $^{21,22,38,39}$

The interdot Coulomb interaction can lead to both the incoherent transfer and hybridization of energy states in the closely packed ensembles of QDs, just as it does in atomic and molecular systems. ${ }^{40}$ The presence or absence of coherence effects in a QD molecule is determined by the relationship between the interdot-interaction matrix element $M_{\mathrm{I}, \mathrm{II}}$ (subscripts I and II correspond to the first and second QDs), energy detuning $\Delta_{\mathrm{I}, \mathrm{II}}=E_{\mathrm{I}}-E_{\mathrm{II}}$ of the QDs' excitations coupled by the interaction, and dephasing rate $\Gamma$ of the interdot transitions. The formation of the entangled states in a pair of QDs is possible when

$$
\left|M_{\mathrm{I}, \mathrm{II}}\right| \gg\left|\Delta_{\mathrm{I}, \mathrm{II}}\right|, \hbar \Gamma \text {. }
$$

Otherwise, only the energy transfer, either reversible or not, can occur. ${ }^{19-21}$

The value of $\Gamma$ can be approximated by a sum of the dephasing rates of electronic transitions in the first and second QDs

$$
\Gamma \approx \Gamma_{\mathrm{I}}+\Gamma_{\mathrm{II}}, \quad \Gamma_{\alpha}=\left(\gamma_{i, \alpha}+\gamma_{f, \alpha}\right) / 2+\bar{\gamma}_{f i, \alpha} \quad(\alpha=\mathrm{I}, \mathrm{II}),
$$

where $\gamma_{i, \alpha}$ and $\gamma_{f, \alpha}$ are the energy relaxation rates of the initial and final transition states and $\bar{\gamma}_{i f, \alpha}$ is the pure dephasing rate of transition $|i, \alpha\rangle \rightarrow|f, \alpha\rangle$. The matrix elements of the dipole-allowed transitions are typically less than a few millielectronvolts in QDs made of direct-bandgap semiconductors, ${ }^{22,39}$ which means that the coherent coupling can only be realized between QDs with relatively slow phase and energy relaxations. A simple estimate of the dephasing rate $\Gamma$ shows 
that the condition in Eq. (1) is satisfied for a pair of identical CdSe QDs at temperatures below $90 \mathrm{~K}$. Since the matrix elements of the dipole-forbidden transitions are much smaller than those of the dipole-allowed one, the forbidden interdot interaction is far less attractive from the viewpoint of experimental investigation of the coherence effects.

It should be noted that one can realize the coherent regime of Coulomb coupling between the QDs of different sizes by applying an electromagnetic field to tune QD's energy spectrum and satisfy the resonance condition. ${ }^{41}$ Such a selective field application is quite a technological challenge, because observation of coherent coupling requires the smallest interdot distance possible.

Another problem associated with the realization of coherent energy transfer in QD systems is the variation of the intraband energy relaxation rates in QDs over a wide range of $10^{9}$ to $10^{13} \mathrm{~s}^{-1}$ even at cryogenic temperatures. ${ }^{42-48}$ Therefore, even if the resonance condition is satisfied for the fundamental transition of one QD and some transition between the high-energy excited states of the other, the coherent coupling may still be absent due to the fast intraband relaxation. We can thus conclude that the coherent coupling should be the easiest to achieve between the lowest-energy states of the QDs of equal sizes, made of a wide-bandgap semiconductor, because the interband relaxation rates of the fundamental transitions in such QDs can be much less than $\left|M_{\mathrm{I}, \mathrm{II}}\right| / \hbar$.

One of the key tasks in the field of nonradiative energy transfer is the development of a theoretical framework of the photoluminescence spectroscopy that would enable distinguishing between different regimes of energy transfer in QD nanostructures and extracting important QD parameters (e.g., energy spectrum and phase relaxation rates) from experimental data. In our previous works, ${ }^{19,21}$ we theoretically studied stationary photoluminescence from double QDs exhibiting the reversible or nonreversible incoherent resonant energy transfer. This paper continues these studies by presenting a theory on the secondary emission from a pair of coherently coupled QDs and analyzing the manifestations of coherence effects in the photoluminescence spectra.

\section{HAMILTONIAN FORMALISM}

Consider a quantum-dot molecule (QDM) whose interaction with classical excitation field and quantum radiation field is described by the Hamiltonian

$$
H=H_{\mathrm{QDM}}+H_{\mathrm{R}}+H_{\mathrm{QDM}, \mathrm{L}}+H_{\mathrm{QDM}, \mathrm{R}},
$$

where the first two terms represent the noninteracting QDM and emitted photons. The terms $H_{\mathrm{QDM}, \mathrm{L}}$ and $H_{\mathrm{QDM}, \mathrm{R}}$ describe the interactions between laser field (L) and vacuum of electromagnetic radiation $(\mathrm{R})$, respectively.

We focus on the QDM composed of two QDs and described by the Hamiltonian

$$
H_{\mathrm{QDM}}=\sum_{\alpha} \sum_{p} E_{p, \alpha} a_{p, \alpha}^{\dagger} a_{p, \alpha}+\sum_{p, q}\left(M_{q \mathrm{I}, p \mathrm{II}} a_{q, \mathrm{I}}^{\dagger} a_{p, \mathrm{II}}+\text { H.c. }\right),
$$

where $E_{p, \alpha}$ is the energy of the electron-hole-pair state $p$ in the first $(\alpha=\mathrm{I})$ or second $(\alpha=\mathrm{II}) \mathrm{QD}$, and $a_{p, \alpha}^{\dagger}$ and $a_{p, \alpha}$ are the creation and annihilation operators of the electron-hole pairs. The matrix element $M_{q \mathrm{I}, p \mathrm{II}} \equiv\left\langle q, \mathrm{I}\left|V_{\mathrm{C}}\right| p, \mathrm{II}\right\rangle$ describes the Coulomb interaction between the QDs, which are assumed to be coupled through the screened potential

$$
V_{\mathrm{C}}\left(\mathbf{r}, \mathbf{r}_{\mathrm{I}}, \mathbf{r}_{\mathrm{II}}\right)=\frac{e^{2}}{\varepsilon\left|\mathbf{r}+\mathbf{r}_{\mathrm{I}}-\mathbf{r}_{\mathrm{II}}\right|},
$$

where $\mathbf{r}$ is the vector directed from the center of the second QD to the center of the first QD, whereas $\mathbf{r}_{I}$ and $\mathbf{r}_{\text {II }}$ are the radius vectors of electrons in the reference frames with the origins at the QD centers. By considering spherical QDs in a dielectric matrix, one can describe the effect of screening using the effective permittivity ${ }^{20,22}$

$$
\varepsilon=\frac{\left(\varepsilon_{\mathrm{I}}+2 \varepsilon_{\mathrm{M}}\right)\left(\varepsilon_{\mathrm{II}}+2 \varepsilon_{\mathrm{M}}\right)}{9 \varepsilon_{\mathrm{M}}},
$$

where $\varepsilon_{\mathrm{I}}, \varepsilon_{\mathrm{II}}$, and $\varepsilon_{\mathrm{M}}$ are the high-frequency permittivities of the QDs and matrix. Note that Eq. (3) neglects the interdot exchange interaction, which is justified due to its weakness for QDMs embedded in dielectric. ${ }^{49}$

The Hamiltonian of noninteracting photons is of the form

$$
H_{\mathrm{R}}=\sum_{k} \hbar \omega_{k} b_{k}^{\dagger} b_{k}
$$

where $b_{k}^{\dagger}$ and $b_{k}$ are the creation and annihilation operators of photons of mode $k$ and frequency $\omega_{k}$, whereas the last two terms in Eq. (2) are given by

$$
H_{\mathrm{QDM}, \mathrm{L}}=\sum_{p, \alpha}\left(\phi(t) V_{p \alpha, 0 \alpha}^{(\mathrm{L})} e^{-\mathrm{i} \omega_{\mathrm{L}} t} a_{p, \alpha}^{\dagger}+\text { H.c. }\right)
$$

and

$$
H_{\mathrm{QDM}, \mathrm{R}}=\sum_{p, \alpha, k} g_{\alpha, k}\left(\mathrm{i} \hbar V_{p \alpha, 0 \alpha}^{(k)} b_{k} a_{p, \alpha}^{\dagger}+\text { H.c. }\right),
$$

where $g_{\alpha, k}=\sqrt{2 \pi \hbar \omega_{k} /\left(\varepsilon_{\alpha} V\right)}, V$ is the normalization volume, $V_{p \alpha, 0 \alpha}^{(\eta)}=-e\left\langle p, \alpha\left|\mathbf{r e}_{\eta}\right| 0, \alpha\right\rangle(\eta=\mathrm{L}, k),-e \mathbf{r}$ is the dipole moment operator, $\mathbf{e}_{\eta}$ is the polarization vector, and $\phi(t)$ is the complex envelope of the excitation field of frequency $\omega_{\mathrm{L}}$.

\section{RESONANT COUPLING OF QUANTUM DOTS}

As was mentioned earlier, the coherent coupling of QDs in a QDM is strongest when the energies of the lowest excited electronic states of the QDs coincide. If this resonance condition is nearly satisfied, then the interdot interaction is dominated by two resonant terms and the Hamiltonian of the QDM takes the form

$$
\begin{aligned}
H_{\mathrm{QDM}}= & E_{\mathrm{I}} a_{\mathrm{I}}^{\dagger} a_{\mathrm{I}}+E_{\mathrm{II}} a_{\mathrm{II}}^{\dagger} a_{\mathrm{II}}+\left\langle 01\left|V_{\mathrm{C}}\right| 10\right\rangle a_{\mathrm{II}}^{\dagger} a_{\mathrm{I}} \\
& +\left\langle 10\left|V_{\mathrm{C}}\right| 01\right\rangle a_{\mathrm{I}}^{\dagger} a_{\mathrm{II}},
\end{aligned}
$$

where $a_{\alpha}^{\dagger}$ and $a_{\alpha}$ are the creation and annihilation operators of the electron-hole pairs in the lowest excited states of energies $E_{\mathrm{I}}$ and $E_{\mathrm{II}}$ and we have employed the following 

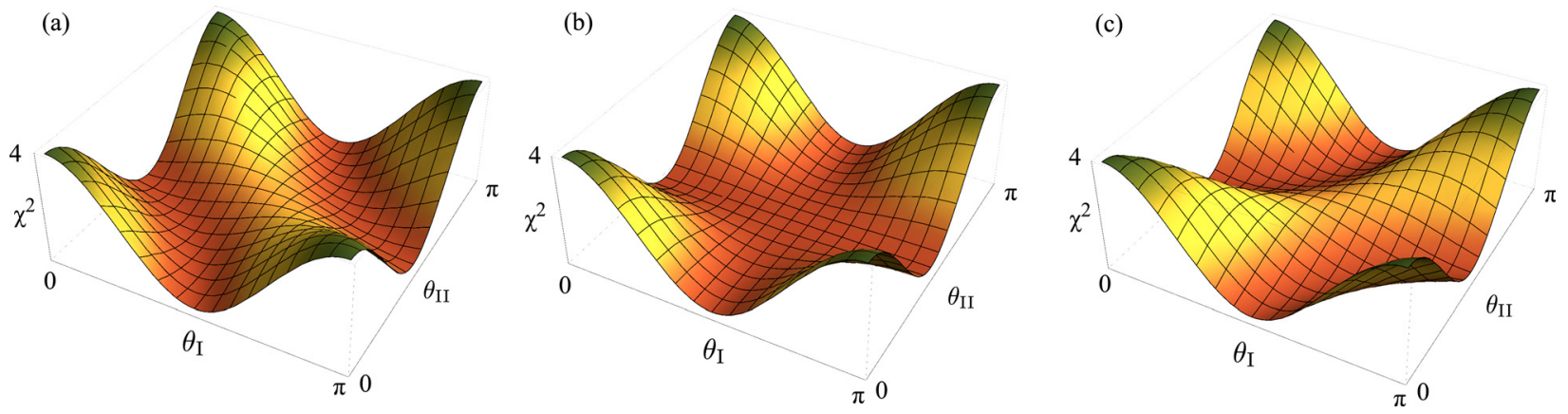

FIG. 1. Dependence of $\chi^{2}$ on polar angles $\theta_{\mathrm{I}}$ and $\theta_{\mathrm{II}}$ for (a) $\varphi=0$, (b) $\varphi=\pi / 2$, and (c) $\varphi=\pi$.

notations for the wave functions of the noninteracting QDs: $|00\rangle=|0, \mathrm{I}\rangle|0, \mathrm{II}\rangle,|10\rangle=|1, \mathrm{I}\rangle|0, \mathrm{II}\rangle$, and $|01\rangle=|0, \mathrm{I}\rangle|1, \mathrm{II}\rangle$.

In order to describe the electronic subsystem of the QDM, we use the approximation of infinitely high potential barriers for the confined electrons and holes and the twoband model of the QDs' band structure. ${ }^{19-21} \mathrm{We}$ also assume that both QDs are in the regime of strong confinement and that their resonant interband transitions are dipole-allowed. Then, the matrix element of the Coulomb potential calculated in the dipole-dipole approximation for the lowest excited state is given by ${ }^{19}$

$$
M_{\mathrm{I}, \mathrm{II}} \equiv\left\langle 10\left|V_{\mathrm{C}}\right| 01\right\rangle=\frac{e^{2} \chi}{\varepsilon r^{3}}\left|\mathbf{r}_{v c}^{(\mathrm{I})}\right|\left|\mathbf{r}_{c v}^{(\mathrm{II})}\right|,
$$

where $\chi$ describes the orientational dependence of $M_{\mathrm{I}, \mathrm{II}}$ and $\left|\mathbf{r}_{c v}^{(\alpha)}\right|=P_{\alpha} / E_{\mathrm{g}}^{(\alpha)}$ is the matrix element of the coordinate operator $\mathbf{r}_{\alpha}$ expressed through the Kane parameter, $P_{\alpha}$, and the bandgap, $E_{\mathrm{g}}^{(\alpha)}$, of the QD's semiconductor. The interband matrix element is of the form $V_{1 \alpha, 0 \alpha}^{(\eta)}=-e \sqrt{2}\left|\mathbf{r}_{c v}^{(\alpha)}\right|$.

By adopting the spherical coordinates with the $z$ axis parallel to vector $\mathbf{r}$, one gets the following functional dependency: ${ }^{50}$

$$
\chi\left(\theta_{\mathrm{I}}, \theta_{\mathrm{II}}, \varphi\right)=\sin \theta_{\mathrm{I}} \sin \theta_{\mathrm{II}} \cos \varphi-2 \cos \theta_{\mathrm{I}} \cos \theta_{\mathrm{II}},
$$

where we have assumed that $\mathbf{r}_{\mathrm{I}}$ and $\mathbf{r}_{\mathrm{II}}$ make angles $\theta_{\mathrm{I}}$ and $\theta_{\mathrm{II}}$ with $\mathbf{r}$, and $\varphi$ is the difference between the azimuths of $\mathbf{r}_{I}$ and $\mathbf{r}_{\mathrm{II}}$. This expression shows that $\chi^{2}$ ranges from zero to four and, thus, significantly affects the energy transfer efficiency. The dependencies of $\chi^{2}$ on the polar angles $\theta_{\mathrm{I}}$ and $\theta_{\mathrm{II}}$ for three different $\varphi$ are plotted in Fig. 1. It can be seen that, regardless of $\varphi, \chi^{2}$ peaks for the following four orientations of the QDs' dipole moments: (i) $\theta_{\mathrm{I}}=\theta_{\mathrm{II}}=0$; (ii) $\theta_{\mathrm{I}}=0$ and $\theta_{\mathrm{II}}=\pi$; (iii) $\theta_{\mathrm{I}}=\pi$ and $\theta_{\mathrm{II}}=0$; and (iv) $\theta_{\mathrm{I}}=\theta_{\mathrm{II}}=\pi$. This implies that the maximum energy transfer efficiency occurs in the QDMs with parallel dipole moments of the QDs.

When the nearly resonant excitations of the two QDs are coupled through the Coulomb potential, they get hybridized and form excitations of the QDM. The states of the new excitations are the superpositions of the QD states and can be easily found via the canonical transformation. ${ }^{51,52}$ Creation of the QDM excitations is described by new operators, $a_{1}^{\dagger}$ and $a_{2}^{\dagger}$, related to the old ones as

$$
\left(\begin{array}{c}
a_{1}^{\dagger} \\
a_{2}^{\dagger}
\end{array}\right)=S_{\vartheta}\left(\begin{array}{c}
a_{\mathrm{I}}^{\dagger} \\
a_{\mathrm{II}}^{\dagger}
\end{array}\right), \quad S_{\vartheta}=\left(\begin{array}{cc}
\cos \vartheta & \sin \vartheta \\
-\sin \vartheta & \cos \vartheta
\end{array}\right),
$$

where the transformation angle $\vartheta=(1 / 2) \arctan \left[2 M_{\mathrm{I}, \mathrm{II}} /\right.$ $\left.\left(E_{\mathrm{I}}-E_{\mathrm{II}}\right)\right](-\pi / 4<\vartheta<\pi / 4)$ is the parameter of canonical transformation. The wave functions and energies of the QDM excitations are given by

$$
\begin{aligned}
|1\rangle\rangle & =\cos \vartheta|10\rangle+\sin \vartheta|01\rangle, \\
|2\rangle\rangle & =\cos \vartheta|01\rangle-\sin \vartheta|10\rangle
\end{aligned}
$$

and

$$
E_{1,2} \equiv \hbar \omega_{1,2}=\frac{1}{2}\left(E_{\mathrm{I}}+E_{\mathrm{II}} \pm \sqrt{\left(E_{\mathrm{I}}-E_{\mathrm{II}}\right)^{2}+4\left|M_{\mathrm{I}, \mathrm{II}}\right|^{2}}\right) .
$$

For the sake of definiteness in the following analysis, we focus on the QDMs with QDs made of the cubic modification of $\mathrm{CdSe}$ or InSb. The material parameters of the two semiconductors are given in Table I. We also assume that the QDMs are embedded in fused silica with a highfrequency permittivity $\varepsilon_{\mathrm{M}}=2.13 .^{54}$

The energy splitting $E_{1}-E_{2}$ of states $\left.|1\rangle\right\rangle$ and $\left.|2\rangle\right\rangle$ is relatively small and very sensitive to the materials and the geometrical parameters of the QDMs. The latter can be concluded from the behavior of the transformation angle $\vartheta$ as a function of a QD radius for a given radius of the other QD and a fixed QDs' spacing illustrated by Fig. 2. As the figure suggests, for hybridization of the QDs' excitations to be efficient, the relative difference of the QDs' radii in a typical QDM cannot exceed 1\%. Since the radii of real QDs vary discretely, with the steps determined by the lattice constants of the QD materials, careful control over the radii, materials, and shapes of the QDM's components is required to achieve the resonance and realize strong coherent coupling even at cryogenic temperatures.

The interaction between the states of the QDM with coherently coupled QDs and the classical excitation field is

TABLE I. Parameters of CdSe and InSb QDs ${ }^{53}$ used in the calculations $\left(m_{c}\right.$ and $m_{v}$ are the effective masses of electrons and holes, respectively; $m_{0}$ is the free-electron mass; and $\hbar \omega_{\mathrm{LO}}$ is the energy of longitudinal optical phonons).

\begin{tabular}{lcccccc}
\hline \hline & $m_{c} / m_{0}$ & $m_{v} / m_{0}$ & $E_{\mathrm{g}}^{(\alpha)}(\mathrm{eV})$ & $P_{\alpha}\left(\mathrm{cm}^{3} \mathrm{~g} \mathrm{~s}^{-2}\right)$ & $\hbar \omega_{\mathrm{LO}}(\mathrm{meV})$ & $\varepsilon_{\alpha}$ \\
\hline $\mathrm{CdSe}$ & 0.11 & 1.14 & 1.736 & $1.30 \times 10^{-19}$ & 26 & 5.8 \\
$\mathrm{InSb}$ & 0.14 & 0.44 & 0.236 & $1.51 \times 10^{-19}$ & 16 & 15.7 \\
\hline \hline
\end{tabular}



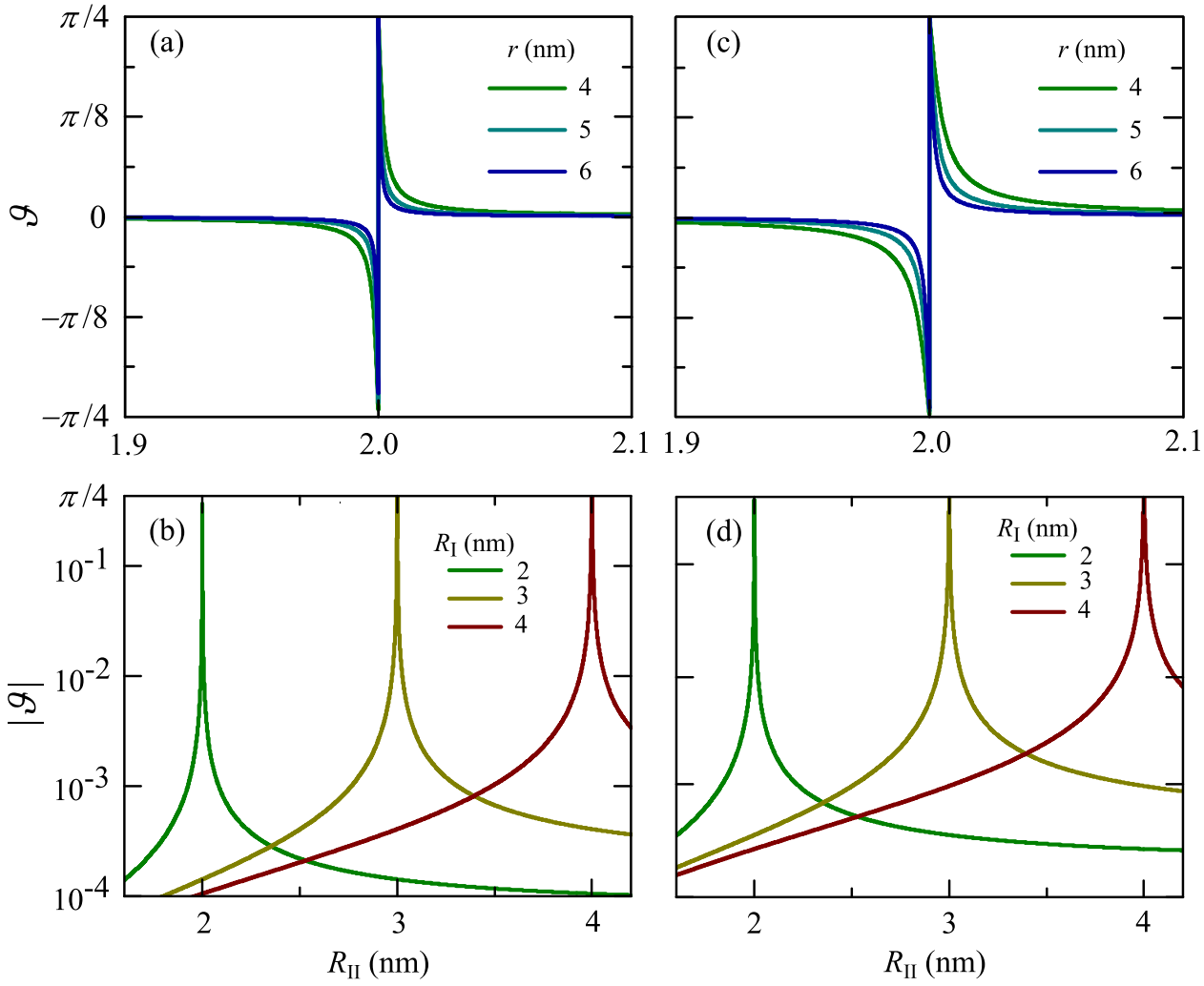

FIG. 2. Transformation angle $\vartheta$ vs radius $R_{\mathrm{II}}$ of the second QD in [(a) and (b)] $\mathrm{CdSe}$ and [(c) and (d)] InSb QDMs; $R_{\mathrm{II}}=2 \mathrm{~nm}$ in (a) and (c); and $r=9 \mathrm{~nm}$ in (b) and (d). The angle jumps at $R_{\mathrm{II}}=R_{\mathrm{I}}$ from $-\pi / 4$ to $\pi / 4$. described by the transformed Hamiltonian $\hat{H}_{\mathrm{QDM}, \mathrm{L}}=\hat{H}_{1 \mathrm{~L}}$ $+\hat{H}_{2 \mathrm{~L}}$, where

$$
\hat{H}_{\beta \mathrm{L}}=\phi(t) e^{-\mathrm{i} \omega_{L} t} V_{1,0}^{(\beta \mathrm{L})} a_{\beta}^{\dagger}+\text { H.c. },
$$

index $\beta$ enumerates the states of the QDM, $|1\rangle\rangle$ and $|2\rangle\rangle$, and the new matrix elements are related to the old ones via

$$
\left(\begin{array}{c}
V_{1,0}^{(1 \mathrm{~L})} \\
V_{1,0}^{(\mathrm{LL})}
\end{array}\right)=S_{\vartheta}\left(\begin{array}{c}
V_{1 \mathrm{I}, 0 \mathrm{I}}^{(\mathrm{L})} \\
V_{1 \mathrm{II}, 0 \mathrm{II}}^{(\mathrm{L})}
\end{array}\right) .
$$

The transformed Hamiltonian of the QDM interaction with the emitted photons of frequencies $\omega_{1 \mathrm{R}}$ and $\omega_{2 \mathrm{R}}$ is also a sum of two terms, $\hat{H}_{\mathrm{QDM}, \mathrm{R}}=\hat{H}_{1 \mathrm{R}}+\hat{H}_{2 \mathrm{R}}$, with

$$
\hat{H}_{\beta \mathrm{R}}=\mathrm{i} B_{\beta} a_{\beta}^{\dagger}-\mathrm{i} B_{\beta}^{\dagger} a_{\beta}
$$

and the new operators are given by

$$
\left(\begin{array}{c}
B_{1} \\
B_{2}
\end{array}\right)=S_{\vartheta}\left(\begin{array}{c}
g_{\mathrm{I}, 1 \mathrm{R}} V_{1 \mathrm{I}, 0 \mathrm{I}}^{(1 \mathrm{R})} b_{1 \mathrm{R}} \\
g_{\mathrm{II}, 2 \mathrm{R}} V_{1 \mathrm{II}, 0 \mathrm{OII}}^{(2 \mathrm{R})} b_{2 \mathrm{R}}
\end{array}\right) .
$$

\section{PHOTOLUMINESCENCE OF A QUANTUM-DOT MOLECULE}

We next use the results of the previous section to calculate the intensity of the photoluminescence from the QDM comprising QDs with resonant electronic subsystems. The energy-level diagram illustrating the excitation of the QDM and its radiative and nonradiative relaxation channels is shown in Fig. 3. Both the interband and intraband nonradiative relaxations, shown by the dashed arrows, occur due to the interaction of the QDM with a bath and are described by the phenomenological rates $\zeta_{01}, \zeta_{02}$, and $\zeta_{21}$. In order to calculate the rates $W_{1}$ and $W_{2}$ of the spontaneous light emission from states $|1\rangle\rangle$ and $|2\rangle\rangle$, we construct a fiveby-five density matrix using the following basis:

$$
\mid 1)=|00\rangle|0 \mathrm{R}\rangle, \quad \mid 2)=|1\rangle\rangle|0 \mathrm{R}\rangle, \quad \mid 3)=|2\rangle\rangle|0 \mathrm{R}\rangle,
$$$$
\mid 4)=|00\rangle|1 \mathrm{R}\rangle, \quad \mid 5)=|00\rangle|2 \mathrm{R}\rangle,
$$

where $|0 R\rangle$ denotes the vacuum of photons and $|1 R\rangle$ and $|2 R\rangle$ are the states of the emitted photons.

The dynamics of the QDM is governed by the generalized master equation for the reduced density matrix ${ }^{55}$

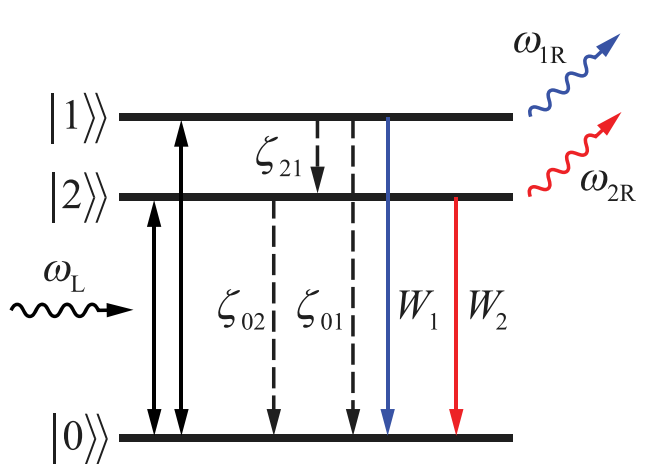

FIG. 3. Energy-level diagram and transitions in a QDM comprising a pair of coherently coupled QDs. The classical optical field of frequency $\omega_{\mathrm{L}}$ excites the low-energy state $|2\rangle\rangle$ and/or the high-energy state $|1\rangle\rangle$ of the QDM. The excited states then decay at rates $\zeta_{01}$ and $\zeta_{02}$ nonradiatively, or at rates $W_{1}$ and $W_{2}$ with the emission of secondary photons $\omega_{1 \mathrm{R}}$ and $\omega_{2 \mathrm{R}}$. Solid and dashed arrows correspond to the radiative and nonradiative transitions, respectively; $\zeta_{21}$ is the rate of transitions $\left.\left.|1\rangle\right\rangle \rightarrow|2\rangle\right\rangle$. 


$$
\frac{\partial \rho_{i j}}{\partial t}=-\frac{\mathrm{i}}{\hbar}[\hat{H}, \hat{\rho}]_{i j}+\delta_{i j} \sum_{k \neq j} \zeta_{j k} \rho_{k k}-\gamma_{i j} \rho_{i j},
$$

where $\delta_{i j}$ is the Kronecker delta, $\zeta_{j k}$ denotes the rate of transitions $|k\rangle\rangle \rightarrow|j\rangle\rangle$ due to the thermal interaction with the bath, $\gamma_{i j}=\left(\gamma_{i i}+\gamma_{j j}\right) / 2+\bar{\gamma}_{i j}$ for $i \neq j$ gives the damping rate of the $\rho_{i j}$ coherence, $\gamma_{i i}$ is the total decay rate of population out of state $\mid i)$, and $\bar{\gamma}_{i j}$ is the pure dephasing rate of transition $\mid j) \rightarrow \mid i)$. Assuming for simplicity that both QDs are made of the same material, one gets the following relaxation operator matrix:

$$
\hat{\gamma}=\left(\begin{array}{ccccc}
0 & \gamma_{01} & \gamma_{02} & 0 & 0 \\
\gamma_{01} & \gamma_{11} & \zeta_{21} & \gamma_{01} & \gamma_{01} \\
\gamma_{02} & \zeta_{21} & \zeta_{02} & \gamma_{02} & \gamma_{02} \\
0 & \gamma_{01} & \gamma_{02} & 0 & 0 \\
0 & \gamma_{01} & \gamma_{02} & 0 & 0
\end{array}\right),
$$

where the rows from top to bottom and columns from left to right correspond to states $\mid 1), \mid 2), \ldots, \mid 5)$, and where $\gamma_{01}=\gamma_{11} / 2+\gamma_{c v}, \gamma_{02}=\zeta_{02} / 2+\gamma_{c v}$, and $\gamma_{11}=\zeta_{01}+\zeta_{21}$.

Parameter $\gamma_{c v}$ can be found from the phenomenological equation for the dephasing rate of interband transitions, ${ }^{19,21}$ and is given by

$$
\gamma_{c v}(T)=\gamma_{0}+a T+b\left[\exp \left(\frac{\hbar \omega_{\mathrm{LO}}}{k_{\mathrm{B}} T}\right)-1\right]^{-1},
$$

where $\gamma_{0}$ is the dephasing rate due to the radiative and nonradiative transitions induced by the interaction with the bath, $\hbar \omega_{\mathrm{LO}}$ is the energy of the longitudinal optical (LO) phonons in QDs, $k_{\mathrm{B}}$ is the Boltzmann constant, and $T$ is the system's temperature. The last two terms in this expression describe the interaction of the QDM with the acoustic and LO phonons through the phenomenological coefficients $a$ and $b$. Since the typical energy splitting of the QDM states due to the interdot Coulomb interaction is of the order of the cutoff energy (a few millielectronvolts) of the acoustic phonon dispersion, ${ }^{56}$ and much smaller than the LO phonon energy (tens of millielectronvolts), the upper state $|1\rangle\rangle$ nonradiatively decays to the lower state $|2\rangle\rangle$ predominantly with the emission of acoustic phonons.

By considering the stationary excitation ( $\phi=$ const) and perturbatively solving Eq. (10) to the lowest orders in the electron-photon interaction, one can find the photon emission rates $W_{1}=\partial \rho_{44} / \partial t$ and $W_{2}=\partial \rho_{55} / \partial t$. The measurable luminescence differential cross section (LDCS), which gives the energy emitted by the QDM per a unit solid angle $\mathrm{d} \Omega$ in a unit frequency interval $\mathrm{d} \omega_{i \mathrm{R}}$, scales in proportion to the photon emission rate and is given by

$$
\frac{\mathrm{d} \sigma_{i}}{\mathrm{~d} \Omega \mathrm{d} \omega_{i \mathrm{R}}}=\frac{V \hbar \omega_{i \mathrm{R}}^{3}}{4(\pi c)^{3}} \frac{W_{i}}{I_{\mathrm{L}}} \quad(i=1 \text { or } 2),
$$

where $I_{\mathrm{L}}$ is the excitation light intensity. With this relationship, the major contributions to the LDCS from the excited states of the QDM are found to be

$$
\begin{aligned}
\frac{\mathrm{d} \sigma_{1}}{\mathrm{~d} \Omega \mathrm{d} \omega_{1 \mathrm{R}}} \approx & C\left(\omega_{1 \mathrm{R}}\right)\left|V_{1 \mathrm{I}, 0 \mathrm{I}}^{(1 \mathrm{R})}\right|^{2}\left[\cos ^{2} \vartheta\left|V_{1,0}^{(1 \mathrm{~L})}\right|^{2} \frac{2}{\gamma_{11}}\right. \\
& \times \frac{\gamma_{01}}{\gamma_{01}^{2}+\Delta_{1,1 \mathrm{R}}^{2} \gamma_{01}^{2}+\Delta_{1, \mathrm{~L}}^{2}}+\sin ^{2} \vartheta \frac{\gamma_{02}}{\gamma_{02}^{2}+\Delta_{2,1 \mathrm{R}}^{2}} \\
& \left.\times \frac{2}{\gamma_{22}}\left(\left|V_{1,0}^{(2 \mathrm{~L})}\right|^{2} \frac{\gamma_{02}}{\gamma_{02}^{2}+\Delta_{2, \mathrm{~L}}^{2}}+\left|V_{1,0}^{(1 \mathrm{~L})}\right|^{2} \frac{\zeta_{21}}{\gamma_{11}} \frac{\gamma_{01}^{2}+\Delta_{01, \mathrm{~L}}^{2}}{\gamma_{01}^{2}}\right)\right]
\end{aligned}
$$

and

$$
\begin{aligned}
\frac{\mathrm{d} \sigma_{2}}{\mathrm{~d} \Omega \mathrm{d} \omega_{2 \mathrm{R}}} \approx & C\left(\omega_{2 \mathrm{R}}\right)\left|V_{1 \mathrm{II}, 0 \mathrm{II}}^{(2 \mathrm{R})}\right|^{2}\left[\sin ^{2} \vartheta\left|V_{1,0}^{(1 \mathrm{~L})}\right|^{2} \frac{2}{\gamma_{11}} \frac{\gamma_{01}}{\gamma_{01}^{2}+\Delta_{1,2 \mathrm{R}}^{2}}\right. \\
& \times \frac{\gamma_{01}}{\gamma_{01}^{2}+\Delta_{1, \mathrm{~L}}^{2}}+\cos ^{2} \vartheta \frac{\gamma_{02}}{\gamma_{02}^{2}+\Delta_{2,2 \mathrm{R}}^{2}} \frac{2}{\gamma_{22}} \\
& \left.\times\left(\left|V_{1,0}^{(2 \mathrm{~L})}\right|^{2} \frac{\gamma_{02}}{\gamma_{02}^{2}+\Delta_{2, \mathrm{~L}}^{2}}+\left|V_{1,0}^{(1 \mathrm{~L})}\right|^{2} \frac{\zeta_{21}}{\gamma_{11}} \frac{\gamma_{01}}{\gamma_{01}^{2}+\Delta_{1, \mathrm{~L}}^{2}}\right)\right],
\end{aligned}
$$

where $C(\omega)=4 \omega^{4} /\left(\pi c^{4} \hbar^{2}\right)$ and $\Delta_{i, j}=\omega_{i}-\omega_{j}$. The intensity of the QDM photoluminescence is the sum of these two contributions.

We illustrate the results obtained by considering two QDMs made of identical QDs: one comprising a pair of 4-nm in diameter CdSe QDs and the other-a pair of 8-nm in diameter InSb QDs (for material parameters refer to Table I). The radii of the QDs were chosen such as to place the luminescence peaks of the QDMs in the visible range $\left(R_{\alpha}=2 \mathrm{~nm}\right.$ and $4 \mathrm{~nm}$ for CdSe and InSb, respectively). According to Eqs. (5), (6), (8), (12a), and (12b), the photoluminescence intensity heavily depends on the mutual orientations of the transition dipole moments and the polarization of the excitation field. For the sake of definiteness, we focus on the ideal situation in which the three vectors $\mathbf{r}_{\mathrm{I}}, \mathbf{r}_{\mathrm{II}}$, and $\mathbf{e}_{\mathrm{L}}$ are codirectional, and thus, both the interdot and the QDM-light interactions are strongest. We also take $T=4 \mathrm{~K}$ and use the following set of phenomenological constants: $\gamma_{0}=7.7 \times 10^{7} \mathrm{~s}^{-1}, \quad a=1.5$ $\times 10^{10} \mathrm{~s}^{-1} \mathrm{~K}^{-1}$, and $b=2.3 \times 10^{13} \mathrm{~s}^{-1},{ }^{21}$ which leads to $\gamma_{c v}=6 \times 10^{10} \mathrm{~s}^{-1}$.

Figure 4 shows how the photoluminescence spectra of the QDMs change with the interdot distance when the excitation energy $\left(\hbar \omega_{\mathrm{L}}=2773.34 \mathrm{meV}\right.$ for CdSe QDs and $\hbar \omega_{\mathrm{L}}=$ $1983.26 \mathrm{meV}$ for InSb QDs) coincides with the fundamental transition energy in the decoupled QDs. The two peaks, centered at frequencies $\omega_{1}$ and $\omega_{2}$, arise in the spectra due to the coherent coupling between the QDs. The peaks are seen to grow with $r$ as they gradually merge together and approach the excitation frequency. The peaks' splitting scales as $1 / r^{3}$ [see Eqs. (5) and (8)], whereas the full width at half maximum (FWHM) of both peaks is about $2 \gamma_{01} \approx 2 \gamma_{02} \approx 80 \mu \mathrm{eV}$ [see Eqs. (12a) with $\vartheta=\pi / 4$ ]. The splitting is slightly larger than $2 \mathrm{meV}$ when the CdSe QDs nearly touch each other $(r=4.5 \mathrm{~nm})$, and becomes too small for experimental resolution when $r$ exceeds $10 \mathrm{~nm}$. In the case of the InSb QDM, the peaks' splitting is about $7 \mathrm{meV}$ for $r=8.5 \mathrm{~nm}$, and the peaks cease to be resolvable only when the interdot distance exceeds $30 \mathrm{~nm}$. 

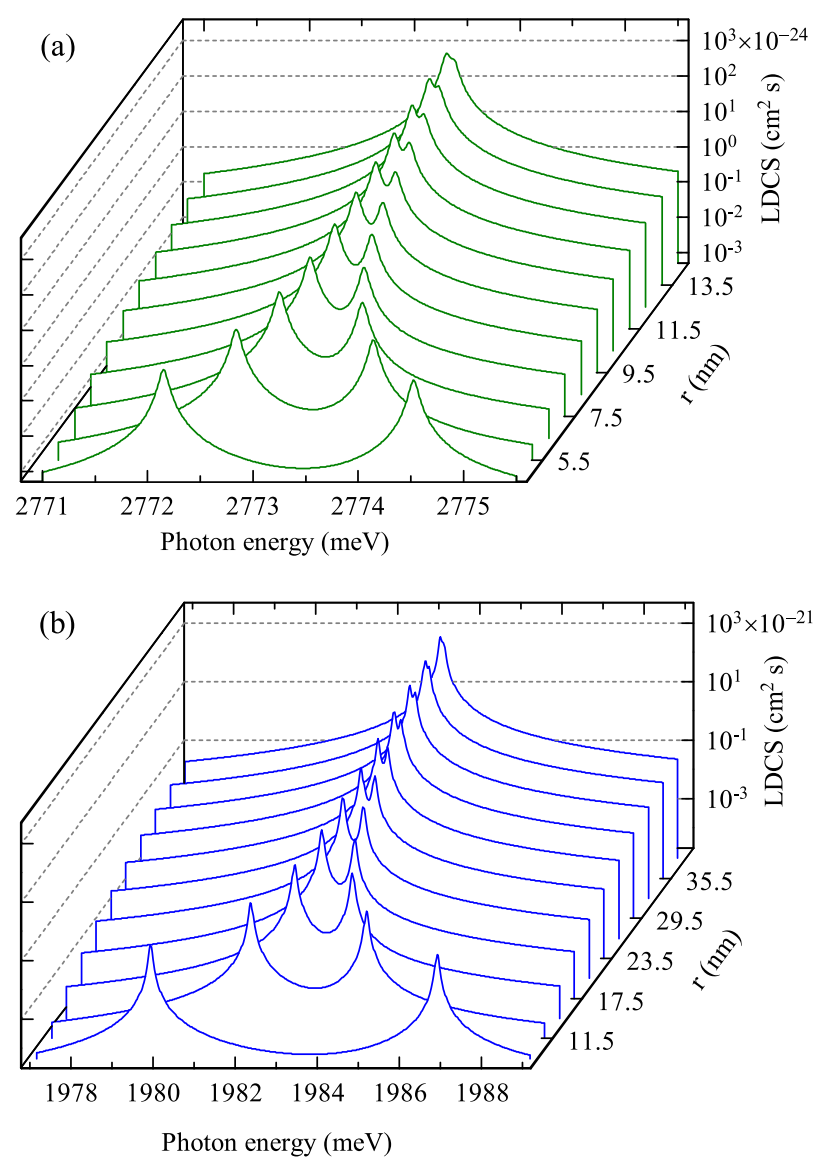

FIG. 4. Photoluminescence spectra of (a) CdSe and (b) InSb QDMs with $\zeta_{01}=\zeta_{02}=\zeta_{12} / 2=10^{8} \mathrm{~s}^{-1}$ for different distances $r$ between the QD centers. The spectra are the sums of LDCSs given in Eqs. (12a) and (12b). For material parameters, refer to the text.

Figure 5 shows the LDCS spectrum of the CdSe QDM with $r=10 \mathrm{~nm}$ and $\zeta_{02}=\zeta_{12} / 2=10^{8} \mathrm{~s}^{-1}$ for three nonradiative relaxation rates from the high-energy molecule's state $|1\rangle\rangle$. Variation of $\zeta_{01}$ is seen to change the intensity of the luminescence peaks while almost not affecting their widths: faster relaxation from state $|1\rangle\rangle$ results in a stronger

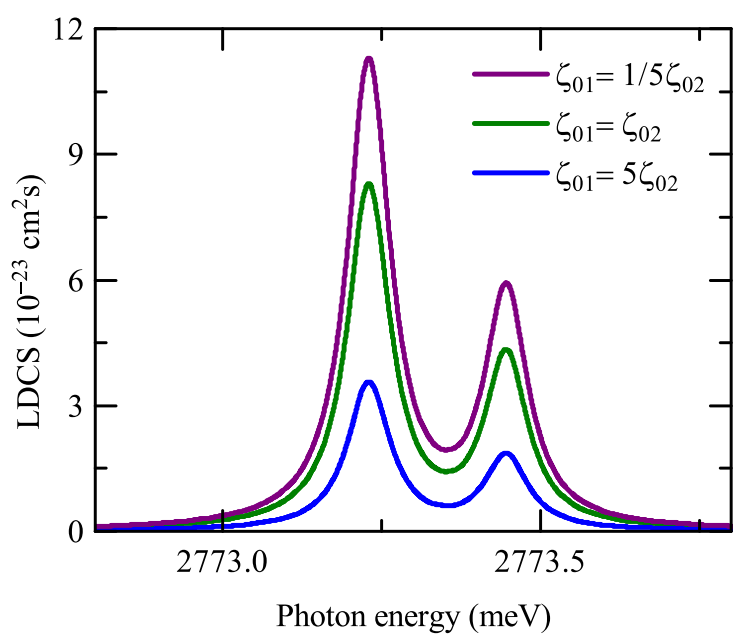

FIG. 5. Photoluminescence spectrum of a CdSe QDM with $r=10 \mathrm{~nm}$ for three nonradiative relaxation rates of state $|1\rangle\rangle$ (see Fig. 3). In all cases, $\zeta_{02}=\zeta_{12} / 2=10^{8} \mathrm{~s}^{-1}$. For other parameters, refer to the text. photoluminescence signal and vise versa. These results demonstrate that by carefully choosing the parameters of the QDs, interdot distance, and excitation frequency, one can tune the positions and relative intensities of the QDM photoluminescence peaks as desired for practical applications.

\section{CONCLUSIONS}

We have developed a theory of low-temperature, stationary photoluminescence from a pair of spherical quantum dots coupled by the Coulomb interaction in a quantum-dot molecule. The lowest-energy electron-hole-pair states of the dots were assumed to be nearly resonant and characterized by low decay and dephasing rates. The coherent coupling of the quantum dots under these conditions was shown to manifest itself in the molecule's photoluminescence spectrum as a pair of peaks, the intensities and spectral positions of which are determined by the geometry and materials of the dots, as well as by the rates of energy and phase relaxations of their electronic subsystems. We also derived an expression for the photoluminescence differential cross section, which is useful for interpreting and analyzing the secondary emission spectra of coherently coupled quantum dots.

\section{ACKNOWLEDGMENTS}

The authors gratefully acknowledge the financial support from the Ministry of Education and Science of the Russian Federation (Grant No. 14.B25.31.0002) and the Russian Foundation for Basic Research (Grants Nos. 12-0201263 and 12-02-00938). The Ministry of Education and Science of the Russian Federation also supports A.S.B. and M.Yu.L., through its scholarships of the President of the Russian Federation for young scientists and graduate students (2013-2015). A.S.B. is also grateful to the Dynasty Foundation Support Program for Physicists. The work of I.D.R. was funded by the Australian Research Council, through its Discovery Early Career Researcher Award No. DE120100055.

${ }^{1}$ S. B. Brichkin, High Energy Chem. 47, 277 (2013).

${ }^{2}$ A. L. Rogach, Nano Today 6, 355 (2011).

${ }^{3}$ T. Unold, K. Mueller, C. Lienau, T. Elsaesser, and A. D. Wieck, Phys. Rev. Lett. 94, 137404 (2005).

${ }^{4}$ G. D. Scholes and D. L. Andrews, Phys. Rev. B 72, 125331 (2005).

${ }^{5}$ K. G. Dvoyan, E. M. Kazaryan, A. A. Tshantshapanyan, Z. M. Wang, and G. J. Salamo, Appl. Phys. Lett. 98, 203109 (2011).

${ }^{6}$ H. V. Demir, S. Nizamoglu, T. Erdem, E. Mutlugun, N. Gaponik, and A. Eychmüller, Nano Today 6, 632 (2011).

${ }^{7}$ J. Basset, D.-D. Jarausch, A. Stockklauser, T. Frey, C. Reichl, W. Wegscheider, T. M. Ihn, K. Ensslin, and A. Wallraff, Phys. Rev. B 88, 125312 (2013).

${ }^{8}$ H. Liu, T. Fujisawa, H. Inokawa, Y. Ono, A. Fujiwara, and Y. Hirayama, Appl. Phys. Lett. 92, 222104 (2008).

${ }^{9}$ J. M. Taylor, H. A. Engel, W. Dür, A. Yacoby, C. M. Marcus, P. Zoller, and M. D. Lukin, Nat. Phys. 1, 177 (2005).

${ }^{10}$ P. Zrazhevskiy, M. Sena, and X. Gao, Chem. Soc. Rev. 39, 4326 (2010).

${ }^{11}$ D. Beljonne, C. Curutchet, G. D. Scholes, and R. J. Silbey, J. Phys. Chem. B 113, 6583 (2009)

${ }^{12}$ T. Förster, Ann. Phys. 437, 55 (1948).

${ }^{13}$ D. L. Dexter, J. Chem. Phys. 21, 836 (1953).

${ }^{14}$ A. P. Litvin, E. V. Ushakova, P. S. Parfenov, A. V. Fedorov, and A. V. Baranov, J. Phys. Chem. C 118, 6531 (2014).

${ }^{15}$ S. Sarkar, A. R. Maity, N. S. Karan, and N. Pradhan, J. Phys. Chem. C 117, 21988 (2013). 
${ }^{16}$ Z. Lin, H. Li, A. Franceschetti, and M. T. Lusk, ACS Nano 6, 4029 (2012).

${ }^{17}$ M. Lunz, A. L. Bradley, V. A. Gerard, S. J. Byrne, Y. K. Gun'ko, V. Lesnyak, and N. Gaponik, Phys. Rev. B 83, 115423 (2011).

${ }^{18}$ P. L. Hernández-Martínez, A. O. Govorov, and H. V. Demir, J. Phys. Chem. C 118, 4951 (2014).

${ }^{19} \mathrm{~S}$. Yu. Kruchinin, A. V. Fedorov, A. V. Baranov, T. S. Perova, and K. Berwick, Phys. Rev. B 81, 245303 (2010).

${ }^{20}$ S. Yu. Kruchinin, A. V. Fedorov, A. V. Baranov, T. S. Perova, and K. Berwick, J. Chem. Phys. 133, 104704 (2010).

${ }^{21}$ S. Yu. Kruchinin, A. V. Fedorov, A. V. Baranov, T. S. Perova, and K. Berwick, Phys. Rev. B 78, 125311 (2008).

${ }^{22}$ G. Allan and C. Delerue, Phys. Rev. B 75, 195311 (2007).

${ }^{23}$ I. D. Rukhlenko and A. V. Fedorov, Opt. Spectrosc. 100, 238 (2006).

${ }^{24}$ I. D. Rukhlenko and A. V. Fedorov, Opt. Spectrosc. 101, 253 (2006).

${ }^{25}$ J. Danckwerts, K. J. Ahn, J. Förstner, and A. Knorr, Phys. Rev. B 73, 165318 (2006).

${ }^{26}$ R. West and S. M. Sadeghi, J. Phys. Chem. C 116, 20496 (2012).

${ }^{27}$ I. D. Rukhlenko, D. Handapangoda, M. Premaratne, A. V. Fedorov, A. V. Baranov, and C. Jagadish, Opt. Express 17, 17570 (2009).

${ }^{28}$ M. Minkov and V. Savona, Phys. Rev. B 87, 125306 (2013).

${ }^{29}$ A. Majumdar, M. Bajcsy, A. Rundquist, E. Kim, and J. Vuckovic, Phys. Rev. B 85, 195301 (2012).

${ }^{30}$ C. Daniel, F. Makereel, L. M. Herz, F. J. M. Hoeben, P. Jonkheijm, A. P. H. J. Schenning, E. W. Meijer, R. H. Friend, and C. Silva, J. Chem. Phys. 123, 084902 (2005).

${ }^{31}$ J. Paloheimo, A. J. Pal, and H. Stubb, J. Appl. Phys. 79, 7800 (1996).

${ }^{32}$ A. S. Baimuratov, I. D. Rukhlenko, V. K. Turkov, A. V. Baranov, and A. V. Fedorov, Sci. Rep. 3, 1727 (2013).

${ }^{33}$ A. S. Baimuratov, I. D. Rukhlenko, and A. V. Fedorov, Opt. Lett. 38, 2259 (2013).

${ }^{34}$ A. Rogach, D. Talapin, E. Shevchenko, A. Kornowski, M. Haase, and H. Weller, Adv. Funct. Mater. 12, 653 (2002)

${ }^{35}$ C. B. Murray, C. R. Kagan, and M. G. Bawendi, Annu. Rev. Mater. Sci. 30, 545 (2000).

${ }^{36}$ A. Sukhanova, Y. Volkov, A. L. Rogach, A. V. Baranov, A. S. Susha, D. Klinov, V. Oleinikov, J. H. M. Cohen, and I. Nabiev, Nanotechnology 18, 185602 (2007).
${ }^{37}$ D.-J. Heijs, V. A. Malyshev, and J. Knoester, J. Chem. Phys. 121, 4884 (2004).

${ }^{38}$ R. Baer and E. Rabani, J. Chem. Phys. 128, 184710 (2008).

${ }^{39}$ C. Curutchet, A. Franceschetti, A. Zunger, and G. D. Scholes, J. Phys. Chem. C 112, 13336 (2008)

${ }^{40}$ V. M. Agranovich and M. D. Galanin, Electronic Excitation Energy Transfer in Condensed Matter (North-Holland, Amsterdam, 1982).

${ }^{41}$ A. Nazir, B. W. Lovett, S. D. Barrett, J. H. Reina, and G. A. D. Briggs, Phys. Rev. B 71, 045334 (2005).

${ }^{42}$ V. K. Turkov, S. Yu. Kruchinin, and A. V. Fedorov, Opt. Spectrosc. 110, 740 (2011).

${ }^{43}$ A. Pandey and P. Guyot-Sionnest, Science 332, 929 (2008).

${ }^{44}$ C. Bonati, A. Cannizzo, D. Tonti, A. Tortschanoff, F. van Mourik, and M. Chergui, Phys. Rev. B 76, 033304 (2007).

${ }^{45}$ E. Hendry, M. Koeberg, F. Wang, H. Zhang, C. de Mello Donega, D. Vanmaekelbergh, and M. Bonn, Phys. Rev. Lett. 96, 057408 (2006).

${ }^{46}$ P. Guyot-Sionnest, B. Wehrenberg, and D. Yu, J. Chem. Phys. 123, 074709 (2005).

${ }^{47}$ A. V. Baranov, A. V. Fedorov, I. D. Rukhlenko, and Y. Masumoto, Phys. Rev. B 68, 205318 (2003)

${ }^{48}$ A. V. Fedorov, A. V. Baranov, I. D. Rukhlenko, and Y. Masumoto, Solid State Commun. 128, 219 (2003).

${ }^{49}$ O. Chikalova-Luzina, D. Samosvat, and G. Zegrya, Tech. Phys. Lett. 40, 350 (2014).

${ }^{50} \mathrm{~V}$. Agranovich and D. Galanin, Electronic Excitation Energy Transfer in Condensed Matter (North-Holland, Amsterdam, 1982).

${ }^{51}$ N. N. Bogoliubov and N. N. Bogoliubov, Jr., Introduction to Quantum Statistical Mechanics (World Scientific, Singapore, 2010).

${ }^{52}$ A. S. Davydov, Quantum Mechanics (Pergamon Press, Oxford, 1976).

${ }^{53}$ Semiconductors, Physics of Group IV Elements and III-V Compounds, 1 st ed., Landolt-Börnstein, New Series, Group III, edited by O. Madelung, M. Schultz, and H. Weiss (Springer-Verlag, Berlin, 1982), Vol. 17, Pt. A.

${ }^{54}$ L. M. Landsberger and W. A. Tiller, Appl. Phys. Lett. 49, 143 (1986).

${ }^{55} \mathrm{~K}$. Blum, Density Matrix Theory and Applications (Plenum Press, New York, 1981).

${ }^{56}$ A. V. Fedorov, A. V. Baranov, and Y. Masumoto, Solid State Commun. 122, 139 (2002). 